\title{
Superspaces and Supersymmetries $\star$
}

\author{
A. Jadczyk ${ }^{1,+}$ and K. Pilch ${ }^{2}$ \\ 1 II. Institut für Theoretische Physik der Universität Hamburg, D-2000 Hamburg, Federal Republic \\ of Germany \\ 2 Institute of Theoretical Physics, University of Wroclaw, 50-205 Wroclaw, Poland
}

\begin{abstract}
A theory of graded Banach modules over a Banach-Grassmann algebra is developed and applied to differential geometry of super-manifolds. The explicit structure of superspaces carrying Poincaré supersymmetry and extended supersymmetry, including central charges, is described.
\end{abstract}

\section{Introduction}

Recently Alice Rogers [1] introduced a concept of supermanifold which seems to have some advantages over previous approaches. The idea is to fix a Grassmann algebra $B_{L}$ (the number of odd generators $L$ being possibly infinite), equip it with a Banach norm, and then work with Banach manifolds exploiting at the same time the Grassmann algebra structure. The present paper is inspired by this idea with the aim of improving a few unsatisfactory aspects of [1] and showing on explicit examples of physical interest how this theory works in practice.

After analysing the mathematical structure involved in [1] we have found that there are only few properties of $B_{L}$ which really matter. Therefore we have introduced a concept of a Banach-Grassmann algebra $Q$ (Sect. 3) which, in general, need not have a denumerable set of odd generators. The most important property of $Q$ (apart of the fact that $Q$ is $Z_{2}$-graded: $Q=Q_{0} \oplus Q_{1}$ ) is its self-duality (see Definition 3.1a). So, we work with the category of graded Banach modules over $Q$, the fundamental principle being that of $Q_{0}$-linearity of all linear maps. Once the category is fixed and fundamental principle taken into account, all the theory becomes simple and quite elegant. In particular the tangent bundle of a supermanifold has exactly the same meaning as in ordinary differential geometry. Tangent vectors are tangent to one-parameter curves, and vector fields generate flows along their integral curves. Appealing to derivations is possible but not necessary. "Odd" vectors are well-defined geometrical objects, and can be constructed at any given point from tangent vectors in a canonical way, their only

\footnotetext{
* Supported in part by the Polish Academy of Science Research Program MR I.1

+ Permanent address: Institute of Theoretical Physics, University of Wroclaw, Cybulskiego 36, 50-205 Wroclaw, Poland
} 
role being that of providing a finite $Q$-module basis if such a basis is, at some step, convenient to use. Section 7, where geometrical structure of supersymmetric superspaces is described, may seem unnecessarily complicated but it was written so as to illustrate this point of view. When we talk there about vertical and horizontal subspaces, they are really two complementary subspaces of the tangent space, one having a finite $Q_{0}$-module basis while the second not. When we talk about principal or affine connections we do mean integral transport along path. And when we discuss symmetries, we mean diffeomorphisms of the manifold which transport fibers into fibers, preserve scalar products, preserve $Q_{0}$-module structure of tangent spaces etc.

We do not discuss problems of supergravity here, but from our discussion of supersymmetric superspaces a local geometrical meaning of supergravity theories should be clear: a curved superspace is a superspace of which each tangent space has exactly the same structure as a typical tangent space of the supersymmetric one. Although we do not touch global questions in this paper (Proposition 5.5 being the only one related to global problems), one should bear in mind that even unquantized supergravity can produce effects which are far from being purely classical ones: there can be regions of superspace where, owing to curvature effects (here by "curvature" we mean a general nonintegrability of vertical and horizontal distributions), a quotient real four-dimensional manifold of General Relativity fails to exist. Equivalently, one can meet singularities in space-time which are not at all singularities in superspace.

\section{Algebraic Preliminaries}

This section consists mostly of definitions and statements which are immediate consequences of these definitions. We deal here with graded Banach modules over graded-commutative Banach algebras. All gradings of this paper are $Z_{2}$ gradings, and we simply use the term "graded" to mean " $Z_{2}$-graded". The indices $r, s$ always run over $Z_{2} \doteq\{0,1\}$, and sums like $r+\ldots+r_{k}$ are always understood $\bmod (2)$.

A Banach space $F$ over $K(K=\mathbb{R}$ or $\mathbb{C})$ is called graded if two complementary subspaces $F_{0}, F_{1} \subset F$ are distinguished so that $F=F_{0} \oplus F_{1}$ as a vector space, and such that the canonical projections $P_{r}: F \rightarrow F_{r}$ are continuous.

For each graded Banach space $F$ the canonical involution $J: F \rightarrow F$ is defined by $J \doteq P_{0}-P_{1}$, so that $a \in F_{r}$ if and only if $J(a)=(-1)^{r} a$.

If $F^{1}, \ldots, F^{p}$ are graded Banach spaces, then the canonical involutions $J^{1}, \ldots J^{p}$ determine the involutive linear map $J \doteq J^{1} \otimes \ldots \otimes J^{p}$ of the algebraic tensor product $F^{1} \odot \ldots \odot F^{p}$. If $F^{\prime}$ is also a graded Banach space, and if $f: F^{1} \times \ldots \times F^{p} \rightarrow F^{\prime}$ is a $p$-linear map, then $f$ can be considered as a linear map $f: F^{1} \odot \ldots \odot F^{p} \rightarrow F^{\prime}$. One defines then two maps $2 f_{r} \doteq f_{r} \doteq f+(-1)^{r} J^{\prime} f J(r=0,1)$, where $J^{\prime}$ is the canonical involution of $F^{\prime}$, so that $f=f_{0}+f_{1}$. The map $f$ is called even (resp. odd) if $f=f_{0}$ (resp. $f=f_{1}$ ). It follows that a $p$-linear map $f$ is even (resp. odd) if and only if

$$
f\left(v_{r_{1}}^{1}, \ldots, v_{r_{p}}^{p}\right) \in F_{r_{1}+\ldots r_{p}+r}^{\prime},
$$


where $v_{r_{i}}^{i} \in F_{r_{l}}^{i}(i=1, \ldots, p)$, and $r=0$ (resp. $\left.r=1\right)$.

A $p$-linear map $f: F \times \ldots \times F \rightarrow F^{\prime}$ is graded symmetric (resp. graded skew-symmetric) if

$$
f\left(v_{r_{1}}^{1}, \ldots, v_{r_{k}}^{k}, v_{r_{k+1}}^{k+1}, \ldots, v_{r_{p}}^{p}\right)=(-)^{r_{k} r_{k+1}+r_{k}} f\left(v_{r_{1}}^{1}, \ldots, v_{r_{k+1}}^{k+1}, v_{r_{k}}^{k}, \ldots, v_{r_{p}}^{p}\right),
$$

where $v_{r_{i}}^{i} \in F_{r_{i}}^{i}(i=1, \ldots, p), k=1, \ldots, p-1$, and $r=0$ (resp. $\left.r=1\right)$.

A graded-commutative Banach algebra is a Banach algebra $Q$ (with, or without unit) which is at the same time a graded Banach space $Q=Q_{0} \oplus Q_{1}$ such that

(i) $a_{r} a_{s}=(-)^{r s} a_{s} a_{r} \in Q_{r+s}, \quad a_{r} \in Q_{r}, a_{s} \in Q_{s}$,

(ii) $\left\|a_{0}+a_{1}\right\|=\left\|a_{0}\right\|+\left\|a_{1}\right\|, \quad a_{r} \in Q_{r}$.

The canonical involution $J$ is then an isometric automorphism of $Q$.

Let $Q$ be a graded-commutative Banach algebra, and let $F$ be a graded Banach space. If $F$ is also a left and right $Q$-module such that

$$
a_{r} v_{s}=(-1)^{r s} v_{s} a_{r} \in F_{r+s}
$$

for $a_{r} \in Q_{r}, v_{s} \in F_{s}$, and if the map $Q \times F \ni(a, v) \mapsto a v \in F$ is continuous, then $F$ is called graded Banach $Q$-module. Observe that if $F$ is initially endowed only with right (resp. left) $Q$-module structure, then the above formula can be considered as a definition of the left (resp. right) action of $Q$ on $F$.

It should be noticed that every $Q$-module can be always considered as a $Q_{0}$-module. The most important $Q_{0}$-modules we shall deal with are

$$
Q_{0}, Q_{1}, Q^{m, n} \doteq\left(Q_{0}\right)^{m} \oplus\left(Q_{1}\right)^{n}, \quad Q^{\bar{m}, \bar{n}} \doteq\left(Q_{1}\right)^{m} \oplus\left(Q_{0}\right)^{n}
$$

The elements of $Q^{m, n}\left(\right.$ resp. $\left.Q^{\bar{m}, n}\right)$ are sequences $\left(a^{A}\right)_{A=1, \ldots, m+n}$, written also as $\left(a^{\mu}, a^{\alpha}\right)_{\mu=1, \ldots, m ; \alpha=1, \ldots, n}$, with $a^{\mu} \in Q_{0}$ and $a^{\alpha} \in Q_{1}$ (resp. $a^{\mu} \in Q_{1}, a^{\alpha} \in Q_{0}$ ). The norm is given by $\|a\| \doteq \sum_{A}\left\|a^{A}\right\|$. The symbol $|A|$ means 0 for $A=\mu, 1$ for $A=\alpha$. The most important graded Banach $Q$-modules are the modules $Q^{m+n}$ graded by

$$
\left(Q^{m+n}\right)_{0} \doteq Q^{m, n}, \quad\left(Q^{m+n}\right)_{1} \doteq Q^{\bar{m}, \bar{n}}
$$

The elements of $Q^{m+n}$ are represented by sequences $\left(a^{A}\right)_{A=1, \ldots, m+n}$ with $a^{A} \in Q$. The group of invertible $Q_{0}$-linear maps $Q^{m, n} \rightarrow Q^{m, n}$ is denoted by $G L(m, n)$.

Let $F^{1}, \ldots, F^{p}, F^{\prime}$ be graded Banach $Q$-modules, and let $f: F^{1} \times \ldots \times F^{p} \rightarrow F^{\prime}$ be a continuous $p$-linear map. Then $f$ is called left $p$ - $Q$-linear if

$$
f\left(v_{r_{1}}^{1}, \ldots, a_{r} v_{r_{k}}^{k}, \ldots, v_{r_{p}}^{p}\right)=(-)^{r\left(r_{1}+\ldots r_{k-1}\right)} a_{r} f\left(v_{r_{1}}^{1}, \ldots, v_{r_{k}}^{k}, \ldots, v_{r_{p}}^{p}\right),
$$

where $v_{r_{i}}^{i} \in F_{r_{i}}^{i}(i=1, \ldots, p), k=1, \ldots, p$, and $a_{r} \in Q_{r}$. The space of all continuous left $p$ - $Q$-linear maps, denoted by $L_{L}\left(F^{1}, \ldots, F^{p} ; F^{\prime}\right)$ and graded

$$
L_{L}\left(F^{1}, \ldots, F^{p} ; F^{\prime}\right)=L_{L}\left(F^{1}, \ldots, F^{p} ; F^{\prime}\right)_{0} \oplus L_{L}\left(F^{1}, \ldots, F^{p} ; F^{\prime}\right)_{1}
$$

according to whether a map is even or odd, is a graded Banach $Q$-module with its $Q$-module structure given by

$$
(f a)\left(v^{1}, \ldots, v^{p}\right) \doteq f\left(v^{1}, \ldots, v^{p}\right) a,
$$

for $a \in Q, v^{i} \in F^{i}(i=1, \ldots, p)$. Similarly the space $L_{R}\left(F^{1}, \ldots, F^{p} ; F^{\prime}\right)$ of all continuous 
right $p$ - $Q$-linear maps $g: F^{1} \times \ldots \times F^{p} \rightarrow F^{\prime}$ is a graded Banach $Q$-module with its $Q$-module structure defined by

$$
(a g)\left(v^{1}, \ldots, v^{p}\right) \doteq a g\left(v^{1}, \ldots, v^{p}\right) .
$$

If $f \in L_{L(R)}\left(F^{1}, \ldots, F^{p} ; F^{\prime}\right)$, then $f^{\prime}$ defined by $\left(f_{r}\right)^{\prime} \doteq\left(J^{\prime}\right)^{r} \circ f$, where $J^{\prime}$ is the canonical involution in $F^{\prime}$, is an element of the space $L_{R(L)}\left(F^{1}, \ldots, F^{p} ; F^{\prime}\right)$. In particular $L_{L}\left(F^{1}, \ldots, F^{p} ; F^{\prime}\right)_{0}=L_{R}\left(F^{1}, \ldots, F^{p} ; F^{\prime}\right) \doteq L_{L R}\left(F^{1}, \ldots, F^{p} ; F^{\prime}\right)_{0}$.

Let $E^{1}, \ldots, E^{p}$ be Banach $Q_{0}$-modules (ungraded), and let $F$ be a Banach graded $Q$-module. Then $L\left(E^{1}, \ldots, E^{p} ; F\right)$ is the graded Banach $Q$-module of all continuous $p-Q_{0}$-linear maps from $E^{1} \times \ldots \times E^{p}$ to $F$. The grading is given by $L\left(E^{1}, \ldots, E^{p} ; F\right)_{r} \doteq L\left(E^{1}, \ldots, E^{p} ; F_{r}\right)$. If $E$ is a Banach $Q_{0}$-module, then the graded Banach $Q$-module $L(E ; Q)$ is denoted by $E^{\dagger}$. If $E, E^{\prime}$ are Banach $Q_{0}$-modules and if $f \in L\left(E ; E^{\prime}\right)$, then $f^{\dagger} \in L_{L R}\left(E^{\prime \dagger} ; E^{\dagger}\right)_{0}$ is defined by

$$
\left(f^{\dagger}(\omega)\right)(v) \doteq \omega(f(v)), \quad \omega \in E^{\prime \dagger}, \quad v \in E .
$$

The $\operatorname{map} f \mapsto f^{\dagger}$ from $L\left(E ; E^{\prime}\right)$ to $L_{L R}\left({E^{\prime \dagger}}^{\prime} ; E^{\dagger}\right)_{0}$ is $Q_{0}$-linear and $\left\|f^{\dagger}\right\| \leqq\|f\|$. The graded Banach $Q$-module $L_{R}\left(E^{\dagger} ; Q\right)$ of all continuous right $Q$-linear maps $w: E^{\dagger} \rightarrow Q$ is denoted by $E^{\dagger \dagger}$. Each element $v \in E$ determines the element $v^{\dagger \dagger} \in E_{0}^{\dagger \dagger}$ given by

$$
v^{\dagger \dagger}(\omega) \doteq \omega(v), \quad \omega \in E^{\dagger} .
$$

The map $v \mapsto v^{\dagger \dagger}$ from $E$ to $E^{\dagger \dagger}{ }_{0}$ is $Q_{0}$-linear and $\left\|v^{\dagger \dagger}\right\| \leqq\|v\|$. The above construction is analogous to that of complexification of a real vector space. One starts there with a real vector space $E$ and considers the space $E^{\dagger}$ of all real linear maps from $E$ to $\mathbb{C}$. The space $E^{\dagger}$ carries then already complex structure, so that $E^{\dagger \dagger}$ is naturally defined as the space of complex linear maps from $E^{\dagger}$ to $\mathbb{C}$.

\section{Banach-Grassmann Algebras}

Let $Q$ be a graded-commutative Banach algebra, and consider $Q$ as a Banach graded $Q$-module. The product map $(a, b) \mapsto a b$ can be then interpreted as a graded symmetric element of $L_{L R}^{2}(Q ; Q)_{0}$. In other words $Q$ is endowed with a canonical, even, $Q$-valued bi- $Q$-linear form. One may therefore ask whether $Q$ is selfdual or not. Selfduality is one of the two requirements each BanachGrassmann algebra should satisfy (see Definition 3.1a and Proposition 3.1 ii). The second important property we require is that the odd elements of $Q$ generate all the algebra except of its scalar part.

Definition 3.1 A graded-commutative Banach algebra $Q$ over $K(K=\mathbb{R}$ or $\mathbb{C})$ is called Banach-Grassmann algebra (BG-algebra, in short) if

(a) For each continuous $Q_{0}$-linear map $f: Q_{r} \rightarrow Q_{s}$ there exists a unique element $u \in Q_{r+s}$ such that $\|u\|=\|f\|$ and $f(a)=a u$ (equivalently, $f(a)=u a$ ) for all $a \in Q_{r}$.

(b) $Q_{0}=K \oplus Q_{0}^{\prime}$, with $\|\lambda+s\|=\|\lambda\|+\|s\|$ for $\lambda \in K, s \in Q_{0}^{\prime}$, where $Q_{0}^{\prime}$ denotes the Banach subalgebra of $Q_{0}$ generated by even powers of elements of $Q_{1}$.

Remark. It follows already from a) that $K \subset Q_{0}$. We do not know to what extent 
a) implies b). One can prove (see [2]) that the graded Banach algebra $B_{\infty}$ constructed in [1] is a BG-algebra.

The following Proposition points out the most important properties of any BG-algebra.

Proposition 3.1 The following properties hold for any BG-algebra $Q$.

(i) For each $a \in Q$,

$\|a\|=\sup \left\{\|a y\|: y \in Q_{1},\|y\|=1\right\}=\sup \left\{\|y a\|: y \in Q_{1},\|y\|=1\right\}$,

in particular if $a y=0$ (resp. $y a=0$ ) for all $y \in Q_{1}$, then $a=0$.

(ii) For each $f \in L_{L}(Q ; Q)_{r}$ (resp. $\left.f \in L_{R}(Q ; Q)_{r}\right)$ there exists a unique element $u \in Q_{r}$ such that

$f(a)=a u($ resp. $f(a)=u a), \quad a \in Q$.

(iii) For each element $a \in Q^{\prime} \doteq Q_{0}^{\prime} \oplus Q_{1}$, and for each $0<\theta<1$, there exists $\alpha \geqq 0$ such that

$\left\|a^{n}\right\| \leqq \alpha \theta^{n} \quad$ for $n=1,2, \ldots$.

(iv) The spectrum of every element $a \in Q$ consists exactly of one point $\sigma(a) \in K$, and the map $\sigma: Q \rightarrow K$ is a unique non-zero character of $Q$.

(v) An element $a \in Q$ is invertible if and only if $\sigma(a) \neq 0$. Then

$a^{-1}=\sigma(a)^{-1} \sum_{n=0}^{\infty}(-1)^{n}(s(a) / \sigma(a))^{n}$,

where $s(a) \doteq a-\sigma(a) \in Q^{\prime}$.

(vi) For each $Q_{0}$-linear, not necessarily continuous, map $f: Q_{1} \rightarrow Q$ one has $\sigma(f(y))=0$ and $f(y) z+f(z) y=0$ for all $y, z \in Q_{1}$.

Proof. (i) is just an explicit expression of the fact that the norm of $f: a \mapsto a u$ is $\|u\|$. To prove (ii) observe that, since $f$ is in particular $Q_{0}$-linear, (a) implies that there exist two elements $\stackrel{0}{u}, \stackrel{1}{u} \in Q_{r}$ such that $f\left(a_{s}\right)=a_{s} \stackrel{s}{u}$ for $a_{s} \in Q_{s}$. But $f$ is assumed to be also left $Q$-linear. Therefore for every $y \in Q_{1}$ we have

$$
y a_{s} \stackrel{s}{u}=y f\left(a_{s}\right)=f\left(y a_{s}\right)=\left(y a_{s}\right)^{s+1} u^{1}=y a_{s}^{s+1},
$$

and (i) implies that $\stackrel{s}{u}={ }^{s} \stackrel{+}{u}$. The property (iii) follows by observing that $Q^{\prime}$ has a dense subspace of nilpotents (owing to b), and then the proof of Lemma $2.7 \mathrm{~b}$ of [1] can be applied verbatim. The map $\sigma: Q \rightarrow K$ is uniquely defined by $a=\sigma(a)+$ $s(a)$, with $\sigma(a) \in K, s(a) \in Q^{\prime}$, and $\sigma$ is evidently a non-zero character of $Q$. It follows that if $a^{-1}$ exists, then $\sigma(a) \neq 0$. Conversely, if $\sigma(a) \neq 0$ then the series in (iv) converges by (iii), and therefore $a^{-1}$ exists. Thus $\sigma(a)$ is the only point of the spectrum of $a$. If $\sigma^{\prime}$ is a continuous multiplicative linear map from $Q$ to $K$, then $\sigma^{\prime}$ annihilates all nilpotents and consequently it annihilates $Q^{\prime}$. Being non-zero it has to coincide with $\sigma$. Finally, if $f: Q_{1} \rightarrow Q$ is a $Q_{0}$-linear map, then for every $y, z, x \in Q_{1}$ we have $f(y) z x=f(y z x)=-f(z) y x$, and (i) implies $f(y) z+f(z) y=0$. In particular $f(y) y=0$. If $\sigma(f(y)) \neq 0$, then by (v) $f(y)$ is invertible and consequently $y=0$ which is a contradiction. Therefore $\sigma(f(y))=0$. 


\section{Vector Superspaces}

Vector superspace is essentially $Q^{m, n}$ (or $Q^{\bar{m}, \bar{n}}$, resp. $Q^{m+n}$ ) with understanding that two elements related by a transformation from $G L(m, n)$ represent the same abstract vector but in two different coordinate systems. Since $Q^{m, n}$ is a $Q_{0}$-module which is not finitely generated, we are forced to use coordinate systems rather than bases. However, as we shall see, a general method of imbedding a vector superspace of the type $Q^{m, n}$ into a vector superspace of the type $Q^{m+n}$ exists. Since $Q^{m+n}$ is finitely generated $Q$-module with $m+n$ generators, a difference between bases and coordinate systems is not an important one. Our reason for investigating vector superspaces of the type $Q^{m, n}$ is of geometrical nature: tangent spaces to supermanifolds are of this type, and although one can always construct the graded tangent space of the type $Q^{m+n}$, the "odd tangent vectors" are not tangent any longer (a more adequate intuition should picture them as being orthogonal to supermanifold). The main objective of this section is to show a mechanism by which every vector superspace acquires its odd counterpart.

Definition 4.1. An $(m, n)$ (resp. $(\bar{m}, \bar{n})$, resp. $(m+n))$-dimensional vector superspace is a pair $(E, \Phi)$, where $E$ is a set and $\Phi$ is a non-empty family of bijections (called coordinate maps) $\Phi \ni \phi: E \rightarrow Q^{m, n}$ (resp. $Q^{\bar{m}, \bar{n}}$, resp. $Q^{m+n}$ ), such that for every pair $\phi, \phi^{\prime} \in \Phi$ we have $\phi^{\prime} \circ \phi^{-1} \in G L(m, n)$. If $(E, \Phi)$ and $(F, \Psi)$ are vector superspaces of the same dimension, then an isomorphism between $(E, \Phi)$ and $(F, \Psi)$ is defined as a pair $(i, j)$ of bijections $i: E \rightarrow F$ and $j: \Phi \rightarrow \Psi$, such that $j(\phi) \circ i=\phi$ for all $\phi \in \Phi$.

The simplest examples of superspaces are $Q^{m, n}, Q^{\bar{m}, \bar{n}}$ and $Q^{m+n}$. They will always be endowed with just one coordinate map - the identity map. In the following the term "vector superspace" will denote a vector superspace of some finite dimension. Observe that a vector superspace of dimension $(\bar{m}, \bar{n})$ can be also considered as having dimension $(n, m)$, and vector superspace of dimension $(m+n)$ can be also thought of as having dimension $(m+n, m+n)$ and a restricted family of coordinate maps. A distinction between the three types is, however, convenient when one deals with different superspaces constructed out of a given one.

If $(E, \Phi)$ is a vector superspace, then $\Phi$ can be uniquely completed to $\Phi^{\prime} \supset \Phi$ so that for each $\Lambda \in G L(m, n)$ there exist $\phi, \phi^{\prime} \in \Phi$ such that $\phi^{\prime} \circ \phi^{-1}=\Lambda$. Although such a completion is always possible, we shall not demand of $\Phi$ to be complete. If $E$ is endowed with an additional geometrical structure (like metric and/or torsion tensor), then natural restrictions on coordinate systems can be imposed. Usually, however, the set $\left\{\phi^{\prime} \circ \phi^{-1}: \phi, \phi^{\prime} \in \Phi\right\}$ is a subgroup of $G L(m, n)$.

Let $(E, \Phi)$ be a vector superspace. Each element $\phi \in \Phi$ induces on $E$ the $Q_{0^{-}}$ module structure: $a v \doteq \phi^{-1}(a \phi(v)), a \in Q_{0}, v \in E$. It also induces the Banach norm $\|v\| \doteq\|\phi(v)\|$. If $\phi$ is replaced with $\phi^{\prime} \in \Phi$, then the induced $Q_{0}$-module structures coincide, and the induced Banach norms are equivalent. In this sense each vector superspace will be considered as a Banach $Q_{0}$-module. If $(E, \Phi)$ and $(F, \Psi)$ are vector superspaces of the same dimension, and if $j: \Phi \rightarrow \Psi$ is a bijection such that $j\left(\phi^{\prime}\right) j(\phi)^{-1}=\phi^{\prime} \phi^{-1}$ for all $\phi, \phi^{\prime} \in \Phi$, then $i \doteq j(\phi)^{-1} \circ \phi$ is independent of $\phi \in \Phi$, and is a $Q_{0}$-linear bicontinuous bijection from $E$ to $F$. The pair $(i, j)$ is then an isomorphism between $(E, \Phi)$ and $(F, \Psi)$.

Let $(E, \Phi)$ be a vector superspace of dimension $(m, n)$ and let $E^{\dagger}$ be the dual of 
the Banach $Q_{0}$-module $E$. Given $\phi \in \Phi$ and $\omega \in E_{r}^{\dagger}$, the map $\omega^{\circ} \phi^{-1}: Q^{m, n} \rightarrow Q_{r}$ is continuous $Q_{0}$-linear. Therefore there exists a unique element $\left(\stackrel{r}{\omega}{ }_{A}\right) \in\left(Q^{m+n}\right)_{r}$ such that

$$
\left(\omega \circ \phi^{-1}\right)(a)=a^{A} \stackrel{r}{\omega}_{A}, \quad a \in Q^{m, n} .
$$

It is evident that the map $\stackrel{r}{\phi}^{\dagger}: \omega \mapsto\left(\stackrel{r}{\omega}_{A}\right)$ from $\left(E^{\dagger}\right)_{r}$ to $\left(Q^{m+n}\right)_{r}$ is an isomorphism of Banach $Q_{0}$-modules. Moreover, given $\phi, \phi^{\prime} \in \Phi$, we have

$$
\stackrel{r}{\phi}^{\dagger} \circ\left(\stackrel{r}{\phi}^{\prime \dagger}\right)^{-1}=\phi^{\prime} \circ \phi^{-1}
$$

on $\left(Q^{m+n}\right)_{r}$. Let $\Phi^{\dagger} \doteq\left\{\stackrel{0}{=} \phi^{\dagger} \oplus \hat{\phi}^{\dagger}: \phi \in \Phi\right)$. Then $\left(E^{\dagger}, \Phi^{\dagger}\right)$ is a vector superspace of dimension $m+n$, and every coordinate map $\phi^{\dagger}: E^{\dagger} \rightarrow Q^{m+n}$ is an isomorphism of graded Banach right $Q$-modules.

We note here the following isomorphisms which follow immediately from Corollary 3.1 and Proposition 3.3.

Proposition 4.1. Let $E, F$ be vector superspaces of dimension $(m, n)$ and $\left(m^{\prime} n^{\prime}\right)$ respectively. Then the following Banach $Q_{0}$-modules are isomorphic (we denote by the same letter $f$ two maps related by the isomorphism).

(i) $L(E ; F) \cong L_{R}\left(F^{\dagger} ; E^{\dagger}\right)_{0} \cong L\left(F_{0}^{\dagger} ; E_{0}^{\dagger}\right)$, the isomorphism being given by

$$
\omega(f(v))=(f(\omega))(\omega), \quad \omega \in F^{\dagger}, \quad v \in E,
$$

(ii) $L^{2}\left(E ; Q_{0}\right) \cong L\left(E ; E^{\dagger}{ }_{0}\right)$, the isomorphism being given by

$$
f(v, w)=(f(w))(v), \quad v, w \in E,
$$

(iii) $L^{2}(E ; F) \cong L\left(F_{0}^{\dagger} ; L\left(E, E_{0}^{\dagger}\right)\right)$, the isomorphism being given by

$$
\omega(f(v, w))=((f(\omega))(w))(v), \quad v, w \in E, \quad \omega \in F_{0}^{\dagger} .
$$

Let $(E, \Phi)$ be a vector superspace of dimension $(m, n)$, and consider the second dual $E^{\dagger \dagger}$ of $E$. Every coordinate map $\phi \in \Phi$ defines the coordinate map $\phi^{\dagger \dagger}: w \mapsto\left(w^{A}\right)$ from $E^{\dagger \dagger}$ to $\left(Q^{m, n}\right)^{\dagger \dagger} \cong Q^{m+n}$ by

$$
w(\omega)=w^{A} \phi^{\dagger}(\omega)_{A}, \quad w \in E^{\dagger \dagger}, \quad \omega \in E^{\dagger} .
$$

If $\phi, \phi^{\prime} \in \Phi$, then $\phi^{\prime \dagger \dagger} \circ \phi^{\dagger \dagger-1}=\phi^{\prime} \circ \phi^{-1}$, and if $v \in E$, then $\phi^{\dagger \dagger}\left(v^{\dagger \dagger}\right)=\phi(v)$. It follows that $E^{\dagger \dagger}=E^{\dagger \dagger} \oplus E^{\dagger \dagger}$ is a superspace of dimension $(m+n)$, and $E$ can be canonically identified with $E^{\dagger \dagger}$. The vector superspace $E^{\dagger \dagger}{ }_{1}$ of dimension $(\bar{m}, \bar{n})$ will be denoted by $\bar{E}$ and called the odd complement of $E$. The second dual $E^{\dagger \dagger}$ understood as $E \oplus \bar{E}$ will be denoted by $\widetilde{E}$.

Proposition 4.2. Let $E$ be a vector superspace of dimension $(m, n)$, and let $\omega \in L\left(E, Q_{r}\right)$. There exists a unique map $\bar{\omega} \in L\left(\bar{E} ; Q_{r+1}\right)$ such that $\bar{\omega} \doteq \omega \oplus \bar{\omega} \in$ $L_{L}(\tilde{E}, Q)_{r}$.

Proof. Choose a coordinate map $\phi$, and identify $E$ with $Q^{m, n}$ and $\bar{E}$ with $Q^{\bar{m}, \bar{n}}$. Then $\omega$ is represented by $\left(\omega_{A}\right) \in\left(Q^{m+n}\right)_{r}$ so that

$$
\omega(v)=v^{A} \omega_{A}, \quad\left(v^{A}\right) \in Q^{m, n} .
$$


Define

$$
\bar{\omega}(\bar{v}) \doteq \bar{v}^{A} \omega_{A}, \quad \bar{v} \in \bar{E} .
$$

It is then easy to see that $\bar{\omega}$ satisfies the statements in the Proposition.

The following important Corollary is an easy twofold generalization of the above result.

Corollary 4.1. Let $E^{1}, \ldots, E^{p}, F$ be vector superspaces, and let $f \in L^{p}\left(E^{1}, \ldots, E^{p} ; F\right)$. For each subset $\kappa \subset\{1, \ldots, p\}$ there exists a unique $\omega_{\kappa} \in L\left(E^{1^{\prime}}, \ldots, E^{p^{\prime}} ; F^{\prime}\right)$ - where $E^{k^{\prime}}=E^{k}$ if $k \in \kappa, E^{k^{\prime}}=\bar{E}^{k}$ if $k \notin \kappa$, and $F^{\prime}=\tilde{F}_{r}$ with $r=\frac{1}{2}\left(1-(-1)^{p-k}\right)$-such that $\tilde{\omega} \doteq \underset{\kappa}{\oplus} \omega_{\kappa} \in L_{L}\left(\tilde{E}^{1}, \ldots, \tilde{E}^{p} ; \tilde{F}\right)_{0}$. If $E^{1}=\ldots=E^{p}$ and $\omega$ is symmetric (resp. skewsymmetric), then $\bar{\omega} \doteq \omega_{\phi}$ is skew-symmetric (resp. symmetric), and $\tilde{\omega}$ is graded symmetric (resp. graded skew-symmetric).

It is important to notice that while a vector superspace $E$ of dimension $(m, n)$, with $n \neq 0$, do not admit a finite $Q_{0}$-module basis, its second dual $\tilde{E}$ is a finitely generated free $Q$-module. Namely, given a coordinate map $\phi: E \rightarrow Q^{m, n}$, the vectors $e_{\mu} \in E(\mu=1, \ldots, m)$ defined by $\phi\left(e_{\mu}\right)^{A}=\delta_{\mu}^{A}$, and the vectors $e_{\alpha} \in \bar{E}(\alpha=1, \ldots, n)$ defined by $\bar{\phi}\left(e_{\alpha}\right)^{A}=\delta_{\alpha}^{A}$, form up a finite (left) $Q$-module basis for $\widetilde{E}$. This fact is an important technical advantage of exploiting the odd complements of vector superspaces.

We shall define now the underlying vector space of a vector superspace. Let $(E, \Phi)$ be a vector superspace of dimension $(m, n)(\operatorname{resp} .(\bar{m}, \bar{n}))$, and let $\varepsilon$ be the closed subspace of $E$ consisting of all vectors $v \in E$ for which $\sigma(\phi(v))=0$ for all (equivalently: for at least one) $\phi \in \Phi$. The quotient space $\hat{E} \doteq E / \varepsilon$ is then an $m$ dimensional (resp. $n$-dimensional) vector space over $K$ called the body of $E$. The canonical projection $E \rightarrow \hat{E}$ will be again denoted by $\sigma$. Each coordinate map $\phi \in \Phi$ defines the isomorphism $\hat{\phi}$ of $\hat{E}$ onto $K^{m}\left(\operatorname{resp} . K^{n}\right)$. If $(E, \Phi)$ and $(F, \Psi)$ are vector superspaces of dimensions $(m, n)$ and $\left(m^{\prime}, n^{\prime}\right)$ respectively, and if $f \in L(E ; F)$, then $\sigma \circ f$ annihilates $\varepsilon$ and therefore defines a linear map $\hat{f}: \hat{E} \rightarrow \hat{F}$ such that $\sigma \circ f=$ $\hat{f} \circ \sigma$. The $K$-linear map $\hat{f}$ is called the even body of $f$. The odd body of $f$ is defined as $\hat{\bar{f}}$, and is a $K$-linear map from $\sigma(\bar{E})$ to $\sigma(\bar{F})$. In coordinates $\hat{f}$ is represented by the matrix $\sigma\left(f_{\mu}^{\mu \prime}\right)$ while $\hat{\bar{f}}$ is represented by the matrix $\sigma\left(f_{\alpha}^{\alpha^{\prime}}\right)$.

\section{Supersmooth Functions}

In this section we introduce the concept of supersmoothness of functions from $Q^{m, n}$ to $Q$. Our discussion of superdifferentiability is simpler than the one given in [1]. In particular, knowing already that a function $f$ is a $C^{\infty}$ map between Banach spaces, we need only to look at its first derivative to know whether $f$ is supersmooth or not, while in [1] an investigation of all derivatives was necessary. Nevertheless the two concepts, ours of supersmoothness, and $G^{\infty}$-differentiability of [1], are equivalent.

We take it for granted that the reader is acquired with elements of differential calculus on Banach spaces (a useful reference text-book being [3]). For the sake of convenience we recall that a function $f: E \rightarrow F$ from a Banach space $E$ 
to another Banach space $F$ is differentiable at $x_{0} \in E$ if there exists a continuous linear map $(D f)\left(x_{0}\right): h \rightarrow(D f)\left(x_{0}\right) \cdot h$ from $E$ to $F$ such that

$$
f\left(x_{0}+h\right)-f\left(x_{0}\right)=(D f)\left(x_{0}\right) \cdot h+0(h),
$$

where $0(h) /\|h\| \rightarrow 0$ with $h \rightarrow 0$. If $f$ is differentiable at every point $x$ in an open neighbourhood of $x_{0}$, then one considers the function $x \mapsto(D f)(x)$ from $E$ to the Banach space $\mathscr{L}(E ; F)$ of all bounded linear operators from $E$ to $F$, and $f$ is said to be twice differentiable at $x_{0}$ if $D f$ is differentiable at $x_{0}$. The second derivative of $f$ at $x_{0}$ is then defined as the first derivative of $D f$ at $x_{0}$ and denoted by $\left(D^{2} f\right)\left(x_{0}\right)$. It follows that $D^{2} f$ is a function from $E$ to $\mathscr{L}(E ; \mathscr{L}(E ; F))$. One then identifies $\mathscr{L}(E ; \mathscr{L}(E ; F))$ with $\mathscr{L}^{2}(E ; F)$ by writing $\left(\left(D^{2} f(a)\right) \cdot h\right) \cdot h^{\prime}=\left(D^{2} f(a)\right) \cdot\left(h, h^{\prime}\right)$ much in the same way as the second derivative of a function $f: \mathbb{R}^{n} \rightarrow \mathbb{R}$ can be considered as a bilinear map from $\mathbb{R}^{n}$ to $\mathbb{R}$ given by

$$
\left(h, h^{\prime}\right) \rightarrow \partial_{\mu \nu} f(a) h^{\mu} h^{\prime \nu} .
$$

If $D^{2} f$ exists and is continuous in a neighbourhood of $x_{0} \in E$, then the bilinear map $D^{2} f\left(x_{0}\right)$ is symmetric (partial derivatives commute). If $D^{n} f(x)$ exists for all $x \in U \subset E$ and $n=1,2, \ldots$, then $f$ is said to be $C^{\infty}(U) . D^{n} f(x)$ is then a symmetric $p$-linear map from $E \times \ldots \times E$ to $F$.

Definition 5.1. Let $Q$ be a BG-algebra, and let $U$ be an open subset of $Q^{m, n}$. A supersmooth function on $U$ is a smooth (i.e. $C^{\infty}$ ) map $f: U \rightarrow Q$ such that $D f(x) \in$ $L\left(Q^{m, n} ; Q\right)$ for all $x \in U$ (i.e. $D f(x)$ is required to be not only linear but also $Q_{0}$ linear).

Observe that if $f$ and $g$ are two supersmooth functions on $U$, then $f+g, f g$, and $J \circ f$ are also supersmooth. These operations make the set of all supersmooth functions into a graded commutative algebra denoted by $Q(U)$. The algebra $Q(U)$ contains $Q$ as the subalgebra of constant functions. Therefore $Q(U)$ is also a graded $Q$-module.

A function $f: U \rightarrow Q^{m^{\prime}, n^{\prime}}$ is called supersmooth if all its components $f^{A^{\prime}}$ : $U \rightarrow Q_{\left|A^{\prime}\right|}$ are supersmooth. The set of all such functions is denoted by $Q\left(U ; Q^{m^{\prime}, n^{\prime}}\right)$ and is an $Q(U)_{0}$-module.

Proposition 5.1. Let $f: U \rightarrow Q$ be a supersmooth function defined on an open subset $U \subset Q^{m, n}$. Then for each integer $p=1,2, \ldots$, and for each $x \in U$, we have $D^{p} f(x) \in L^{p}\left(Q^{m, n} ; Q\right)$.

Proof. We prove it by induction. Suppose we already know that $D^{p-1} f(x)$ is in $L^{p-1}\left(Q^{m, n} ; Q\right)$ for all $x \in U$. Then, given $x_{0} \in U$, we have $D^{p} f\left(x_{0}\right)=D\left(D^{p-1} f\right)\left(x_{0}\right)$, and therefore $D^{p} f\left(x_{0}\right)$ is a continuous linear map from $Q^{m, n}$ to $L^{p-1}\left(Q^{m, n} ; Q\right)$. Interpreted as a $p$-linear map from $Q^{m, n}$ to $Q$ it is $Q_{0}$-linear with respect to $p-1$ variables. Since $D^{p} f\left(x_{0}\right)$ is symmetric, it follows that it is automatically $Q_{0}$-linear in all of its $p$ variables.

Definition 5.2. Let $f \in Q(U)$ and $x \in U \subset Q^{m, n}$. We already know that $D^{p} f(x) \in$ $L^{p}\left(Q^{m, n} ; Q\right)$ and therefore (Proposition 3.2) there exists a unique family $u_{A_{1} \ldots A_{p}}(x)$ of elements of $Q$ such that

$$
D^{p} f(x)\left(t_{1}, \ldots, t_{p}\right)=t_{p}^{A_{p}} \ldots t_{1}^{A_{1}} u_{A_{1} \ldots A_{p}}(x)
$$


for all $t_{i}=\left(t_{i}{ }^{A}\right) \in Q^{m, n}(i=1,2, \ldots, p)$. We define then

$$
\frac{\partial^{p} f(x)}{\partial x^{A_{1} \ldots} \partial x^{A_{p}}} \doteq u_{A_{1} \ldots A_{p}}(x) .
$$

Proposition 5.2. If $f \in Q(U)$ then the functions

$$
\partial_{A_{1} \ldots A_{p}} f: x \mapsto \frac{\partial^{p} f(x)}{\partial x^{A_{1} \cdots} \partial x^{A_{p}}}
$$

are also in $Q(U)$, and

$$
\partial_{A_{1} \ldots A_{p}} f=\partial_{A_{1}}\left(\partial_{A_{2} \ldots A_{p}} f\right)
$$

Moreover

$$
\partial_{A_{1} \ldots A_{k} A_{k+1} \ldots A_{p}} f=(-1)^{\left|A_{k}\right|\left|A_{k+1}\right|} \partial_{A_{1} \ldots A_{k+1} A_{k} \ldots A_{p}} f .
$$

Proof. With $A_{1}, \ldots, A_{p}$ fixed, the map $g \rightarrow g_{A_{1} \ldots A_{p}}$ from $L^{p}\left(Q^{m, n} ; Q\right)$ to $Q$ defined in Proposition 3.2 is $Q_{0}$-linear and continuous. Therefore the function $\partial_{A_{1} \ldots A_{p}} f$ is also supersmooth. Then the statement follows by a standard application of the theorem on differentiability of compositions of differentiable mappings of Banach spaces. The last formula is a direct consequence of commutativity of partial derivatives.

We note at this place a lemma which will be useful in the next section.

Lemma 5.1. Let $U \subset Q^{m, n}$ be an open ball, and let $x_{0} \in U$. For every function $f \in Q(U)$ there exist functions $g_{A} \in Q(U)(A=1, \ldots, m+n)$ such that

$$
\begin{aligned}
& f(x)=f\left(x_{0}\right)+\left(x^{A}-x_{0}^{A}\right) g_{A}(x) \\
& g_{A}\left(x_{0}\right)=\left(\partial_{A} f\right)\left(x_{0}\right)
\end{aligned}
$$

Proof. Define

$$
g_{A}(x)=\int_{0}^{1}\left(\partial_{A} f\right)\left(x_{0}+t\left(x-x_{0}\right)\right) d t,
$$

and apply the Taylor's Theorem [3].

Suppose now $f$ is a supersmooth function from $Q^{m, n}$ to $Q^{m^{\prime}, n^{\prime}}$, i.e. all the components $f^{A}: Q^{m, n} \rightarrow Q_{\left|A^{\prime}\right|}$ are supersmooth. Then

$$
f_{A}^{B^{\prime}}(x) \doteq \partial_{A} f^{B^{\prime}}(x)
$$

is a matrix with entries $f_{A}{ }^{B^{\prime}}(x) \in Q_{|A|+\left|B^{\prime}\right|}$. If $g: Q^{m^{\prime}, n^{\prime}} \rightarrow Q^{m^{\prime \prime}, n^{\prime \prime}}$ is also supersmooth then

$$
(g \circ f)_{A}^{C^{\prime \prime}}=f_{A}{ }^{B^{\prime}} g_{B^{\prime}}{ }^{C^{\prime \prime}}
$$

In particular, when $m^{\prime}=m, n^{\prime}=n$ and $f^{-1}$ exists and is supersmooth, then

$$
f_{A}{ }^{B^{\prime}}\left(f^{-1}\right)_{B^{\prime}}{ }^{C}=\delta_{A}^{C} \text {. }
$$

Proposition 5.3. (Inverse function theorem) Let $V \subset Q^{m, n}$ be an open set, and let $f \in Q\left(V, Q^{m, n}\right)$. Suppose the matrix $\sigma\left(f_{A}{ }^{B}\left(x_{0}\right)\right)$ is invertible for some $x_{0} \in V$. 
Then there exists an open neighbourhood $U \subset V$ of $x_{0}$ such that the restriction of $f$ to $U$ is a homeomorphism of $U$ onto an open neighbourhood of $y_{0}=f\left(x_{0}\right)$. Furthermore the inverse mapping $g$ of $f(U)$ onto $U$ is also supersmooth.

Proof. By Corollary 3.1 the map $(D f)\left(x_{0}\right)$ is invertible and therefore the standard inverse function Theorem [3] implies that $(D f)(x)$ is invertible for $x \in U$, that $g \in C^{\infty}(f(U) ; U)$ exists, and $(D f(x)) \circ(D g(f(x)))=i d_{U}(x)$. Then $(D g)(y)$, being the inverse of a $Q_{0}$-linear map $D f(x)$, is also $Q_{0}$-linear, and therefore $g$ is also supersmooth.

The rest of this section deals with the problem of natural domains of definition of supersmooth functions and the following results, although important for clarifying the structure of the sheaf $U \rightarrow Q\left(U ; Q^{m^{\prime}, n^{\prime}}\right)$ of supersmooth maps, will not be used in Sects. 6 and 7.

Definition 5.3. For each subset $W \subset Q^{m, n}$ let $W^{-}$denotes the set $\sigma^{-1}(\sigma(W))$ i.e.

$$
W^{-} \doteq \prod_{A=1}^{m}\left\{\sigma^{-1}\left(\sigma\left(\operatorname{pr}_{A}(W)\right)\right\} \times\left(Q_{1}\right)^{n}\right.
$$

A set $W \subset Q^{m, n}$ is called $\sigma$-convex (resp. $\sigma$-connected) if for each $a \in W$ the set $\{a\}^{-} \cap W$ is convex (resp. connected).

Proposition 5.4. Let $U \subset Q^{m, n}$ be open, $\sigma$-connected, and let $f \in Q\left(U ; Q^{m^{\prime}, n^{\prime}}\right)$. Then

(i) if $a, a^{\prime} \in U$ and $\sigma(a)=\sigma\left(a^{\prime}\right)$, then $\sigma(f(a))=\sigma\left(f\left(a^{\prime}\right)\right)$

(ii) $f$ admits a unique extension $f^{-} \in Q\left(U^{-} ; Q^{m^{\prime}, n^{\prime}}\right)$

(iii) $\sigma\left(f^{-}\left(U^{-}\right)\right)=\sigma(f(U))$.

To prove that above Proposition we shall first establish two Lemmas.

Lemma 5.2. Let $U \subset Q^{m, n}$ be an open set. Then for each $a \in U^{-}$there exists $c \in U$ such that $h^{A} \doteq a^{A}-c^{A}$ are nilpotents for $A=1, \ldots, m+n$.

Proof. If $a \in U^{-}$then there exists $b \in U$ such that $\sigma\left(a^{A}\right)=\sigma\left(b^{A}\right)(A=1, \ldots, m+n)$. Let $\delta>0$ be such that the open ball centered at $b$ and with radius $\delta$ is contained in $U$. Since $a^{A}-b^{A} \in Q_{0}^{\prime}$ for $A=1, \ldots, m$, it follows that there exist nilpotents $h^{A} \in Q_{0}$ such that $\left\|a^{A}-b^{A}-h^{A}\right\|<\delta / m$. Let $c^{A}=a^{A} \dot{-} h^{A}$ for $A=1, \ldots, m$, and $c^{A} \doteq b^{A}$ for $A=m+1, \ldots, m+n$. Since $\|c-b\|<\delta$ it follows that $c \in U$.

Lemma 5.3. Let $U \subset Q^{m, n}$ be open $\sigma$-convex, and let $f \in Q\left(U ; Q^{m^{\prime}, n^{\prime}}\right)$. Then $f$ admits a unique extension $f^{-} \in Q\left(U^{-} ; Q^{m^{\prime}, n^{\prime}}\right)$. If $V \subset U$ is also open and $\sigma$-convex, then $(f \mid V)^{-}=f^{-} \mid V^{-}$. Moreover $\sigma\left(f^{-}\left(U^{-}\right)\right)=\sigma(f(U))$.

Proof. Take any $a \in U^{-}$and let $h^{A}(A=1, \ldots, m+n)$ be nilpotents such that $c \doteq a-h \in U$. Let

$$
f_{h}^{-}(a) \doteq \sum_{p}(1 / p !) h^{A_{1}} \ldots h^{A_{p}}\left(\partial_{A_{p} . . A_{1}} f\right)(a-h),
$$

the series being finite since $h^{A}$ are nilpotents. It is easy to see (compare proof of the Proposition 2.11.c in [1]) that $f_{h}^{-}(a)$ is independent of a choice of nilpotents 
$h^{A}$ as long as $a-h \in U$. Therefore, with $h$ fixed, the above formula defines $f^{-}(\cdot)$ in a neighbourhood of $a$. It follows that $f^{-}$is supersmooth. Any other supersmooth extension of $f$ coincides with $f^{-}$owing to the Taylor formula and $\sigma$ convexivity of $U$. From the uniqueness of the above extension it easy follows that the extension and restriction maps commute. Finally if $a \in U$ then $f^{-}(a)=$ $f_{h}^{-}(a)$ and therefore $\sigma\left(f^{-}(a)\right)=\sigma\left(f_{h}^{-}(a)\right)=\sigma(f(a-h)) \in \sigma(f(U))$.

Proof of the Proposition 5.4. For each $a \in U$ let $U(a) \subset U$ be an open ball centered at $a$, and let, for each $b \in U^{-}, V(b) \doteq\left\{a \in U: b \in U(a)^{-}\right\}$. With $b \in U^{-}$fixed we shall first show that the map $a \mapsto(f \mid U(a))^{-}(b)$ is constant on $V(b)$. Indeed, suppose $a, a^{\prime} \in V(b)$ i.e. there exist $c \in U(a), c^{\prime} \in U\left(a^{\prime}\right)$ such that $\sigma(c)=\sigma\left(c^{\prime}\right)=\sigma(b)$. Then, since $c \in U(a) \cap U(c)$, it follows that $b \in(U(a) \cap U(c))^{-}$. But (Lemma 5.3.) $(f \mid U(a))^{-}\left|(U(a) \cap U(c))^{-}=(f \mid U(a) \cap U(c))^{-}=(f \mid U(c))^{-}\right|(U(a) \cap U(c))^{-}$, and so $(f \mid U(a))^{-}(b)=(f \mid U(c))^{-}(b)$. Similarly $\left(f \mid U\left(a^{\prime}\right)\right)^{-}(b)=\left(f \mid U\left(c^{\prime}\right)\right)^{-}(b)$. We have therefore to show that $(f \mid U(c))^{-}(b)=\left(f \mid U\left(c^{\prime}\right)\right)^{-}(b)$. The set $b^{-} \cap U$ is connected and $\left\{b^{-} \cap U(a): a \in V(b)\right\}$ is its open covering. Therefore there exist $c_{1}, \ldots, c_{k} \in V(b)$ such that $c_{1}=c, c_{k}=c^{\prime}$ and $U\left(c_{i}\right) \cap U\left(c_{i+1}\right) \neq \varnothing$. But then $\left(f \mid U\left(c_{1}\right)\right)^{-}(b)=$ $\left.\left(\left(f \mid U\left(c_{1}\right)\right)^{-} \mid U\left(c_{1}\right) \cap U\left(c_{2}\right)\right)^{-}\right)(b)=\left(f \mid U\left(c_{2}\right)\right)^{-}(b)=\ldots=\left(f \mid U\left(c_{k}\right)\right)^{-}(b)$. In this way we have defined $f^{-}(b)$ as $(f \mid U(a))^{-}(b)$ for some $a \in V(b)$. It is evident that $f^{-}$ so defined is supersmooth since $(f \mid U(a))^{-}$defines not only $f^{-}(b)$ but also $f^{-}\left(b^{\prime}\right)$ for all $b^{\prime} \in U(a)^{-}$. Let now $g$ be some other supersmooth extension of $f$. Then $g(b)=(g \mid U(a))^{-}(b)=(f \mid U(a))^{-}(b)=f^{-}(b)$, and therefore $f^{-}$is unique. We also have $\sigma\left(f^{-}(b)\right)=\sigma\left((f \mid U(a))^{-}(b) \subset \sigma(f(U(a)))\right.$ by Lemma 5.3. It remains to show that for $a, a^{\prime} \in U$ such that $\sigma(a)=\sigma\left(a^{\prime}\right)$ we have $\sigma(f(a))=\sigma\left(f\left(a^{\prime}\right)\right)$. Observe that the segment $\left\{t a+\left(1-t a^{\prime}: 0 \leqq t \leqq 1\right\}\right.$ is contained in $U^{-}$. Then by Lemma 5.1.,

$$
f\left(a^{\prime}\right)=f^{-}\left(a^{\prime}\right)=f^{-}(a)+\left(a^{\prime}-a\right)^{A} g_{A}\left(a^{\prime}\right)=f(a)+\left(a^{\prime}-a\right)^{A} g_{A}(a),
$$

and therefore $\sigma\left(f\left(a^{\prime}\right)\right)=\sigma(f(a))$.

It follows from the above result that a natural domain of definition for a supersmooth function $f: Q^{m, n} \rightarrow Q$ is a set of the form $\sigma^{-1}(U)$, where $U$ is open in $\mathbb{R}^{m}$. If $f$ is defined on such a set, then by the Taylor's Theorem there exist uniquely defined supersmooth functions $f_{\alpha_{1}, \ldots \alpha_{p}}(x)$ such that

$$
f\left(x^{\mu}, \theta^{\alpha}\right)=\sum_{p=0}^{n} \frac{1}{p !} \theta^{\alpha_{1}} \ldots \theta^{\alpha_{p}} f_{\alpha_{p}, \ldots, \alpha_{1}}\left(x^{\mu}\right),
$$

with $f_{\alpha_{1}, \ldots \alpha_{1}, \alpha_{1}+1, \ldots, \alpha_{p}}=-f_{\alpha_{1}, \ldots, \alpha_{l}+1, \alpha_{1}, \ldots, \alpha_{p}}$. Therefore a dependence of $f$ on its odd arguments is rather trivial. It is more difficult to investigate analyticity properties with respect to the even arguments. Suppose $f: Q^{m, 0} \rightarrow Q$ is supersmooth, defined on $\sigma^{-1}(U) \subset Q^{m, 0}, U \subset \mathbb{R}^{m}$, and let $\hat{f}$ denotes the restriction of $f$ to $U \subset \mathbb{R}^{m} \subset Q^{m, 0}$. It is easy to see that $\left(\hat{\partial}_{\mu_{1} \ldots \mu_{p}} f\right)^{-}=\partial_{\mu_{1} \ldots \mu_{p}} \hat{f}$, where the derivatives on the right-hand side are with respect to $m$ real variables. In particular $\hat{f} \in C^{\infty}(U)$. It is also easy to prove that the map $f \mapsto \hat{f}$ from $Q\left(\sigma^{-1}(U) ; Q\right)$ to $C^{\infty}(U ; Q)$ is injective. Namely, if $\hat{f} \equiv 0$, then $\left(\partial_{\mu_{1}, \ldots, \mu_{p}} f\right)^{\wedge}=\partial_{\mu_{1}, \ldots, \mu_{p}} \hat{f} \equiv 0$. Let $a^{\mu}(\mu=1, \ldots, m)$ be nilpotents and let $\lambda \in U$. Then $\lambda+t a \in \sigma^{-1}(U)$ and by the Taylor's Theorem

$$
f(\lambda+a)=\hat{f}(\lambda)+a^{\mu} \hat{\partial}_{\mu} \hat{f}(\lambda)+\ldots+(1 / N !) a^{\mu_{1}} \ldots a^{\mu_{p}}\left(\partial_{\mu_{p} \ldots \mu_{1}} \hat{f}\right)(\lambda)=0 .
$$


Therefore $f$ vanishes on a dense subset of $\sigma^{-1}(\lambda)$. It follows that $f \equiv 0$ on $\sigma^{-1}(U)$. One can show that the map $f \rightarrow \hat{f}$ is not a surjection onto $C^{\infty}(U ; Q)$. On the other hand an analytic function can be always extended from an open ball $U \subset \mathbb{R}^{m}$ to $\sigma^{-1}(U) \subset Q^{m, 0}$. A precise characterization of the range of the map $f \mapsto \hat{f}$ is however not known.

\section{Supermanifolds}

Our definition of a supermanifold (apart of the fact that we allow $Q$ to be an arbitrary BG-algebra) is equivalent to that given in [1]. What differs the two approaches is a treatment of tangent bundles. We define tangent space in quite a conventional way and then apply the extension procedure developed in Sect. 4. The reader is assumed here to be familiar with elements of the theory of Banach manifolds, the recommended reference being [4].

Definition 6.1. A supermanifold is a Banach manifold $M$ modelled on $Q^{m, n}$, and with a supersmooth atlas $\Phi$ of local coordinate maps $\phi$. For each open set $U \subset M$ we denote by $Q(U)$ the graded algebra of supersmooth functions on $U$.

Given a point $p \in M$; let $c, c^{\prime}$ be two $C^{1}$ maps from an open neighbourhood of $0 \in \mathbb{R}$ to $M$, such that $c(0)=c^{\prime}(0)=p$. We call $c$ and $c^{\prime}$ equivalent if for each (equivalently, for some) coordinate system $\phi$ around $p$, the functions $\phi \circ c$ and $\phi \circ c^{\prime}$ have the same derivative at $0 \in \mathbb{R}$. The equivalence classes for this relation are called tangent vectors at $p$, and the set of all tangent vectors at $p$ is denoted by $T(M ; p)$. Each coordinate system $\phi$ defined around $p$ defines the bijection $\phi: T(M ; p) \rightarrow Q^{m, n}$ which sends every tangent vector $v=[f]$ from $T(M ; p)$ into the element $\left(v^{A}\right) \doteq$ $\left.(d / d t)(\phi \circ f)\right|_{t=0}$ of $Q^{m, n}$. When $\phi=\left(x^{A}\right)$ is replaced by $\phi^{\prime}=\left(x^{A^{\prime}}\right)$ then

$$
v^{A^{\prime}}=v^{A} \frac{\partial x^{A^{\prime}}}{\partial x^{A}}(x(p)) .
$$

It follows that $T(M ; p)$ is an $(m, n)$-dimensional vector superspace, so that one can apply the results of Sect. 4 and construct the odd complement $\bar{T}(M ; p)$ of $T(M ; p)$, and the graded Banach left $Q$-module $\tilde{T}(M ; p)=T(M ; p) \oplus \bar{T}(M ; p)$. By the standard methods of differential geometry we construct then the vector bundles $T(M), \bar{T}(M)$ and $\tilde{T}(M)$, which are supermanifolds with supersmooth projections onto $M$. A graded vector superfield $X$ is defined as a supersmooth section of the bundle $T(M)$. Each graded vector superfield is then the sum $X=$ $X_{0}+X_{1}$ of its even and odd part which are supersmooth sections of the bundles $T(M)$ and $\bar{T}(M)$ respectively. An obviously equivalent definition of a graded tangent vector is the following one: a graded tangent vector $v \in T(M ; p)$ is a geometrical object $v$ defined in each coordinate system $\phi=\left(x^{A}\right)$ around $p$ by its components $\left(v^{A}\right) \in Q^{m+n}$ which transform according to the formula (6.1). Then $v$ is even (resp. odd) if $\left(v^{A}\right) \in Q^{m, n}$ (resp. $\left.Q^{\bar{m}, \bar{n}}\right)$. What is not obvious is that a graded tangent vector can be also defined as a derivation of the graded algebra of germs of supersmooth functions at $p$. We shall proceed now to prove this important fact.

Given $p \in M$ let $Q_{p}(M)$ be the graded algebra of germs of supersmooth functions 
at $p$. An even (resp. odd) derivation at $p$ is an even (resp.odd) $Q_{0}$-linear map $v: Q_{p}(M) \rightarrow Q$ such that

$$
v(f g)=v(f) g(p)+J^{|v|}(f(p)) v(g),
$$

where $|v|=0$ (resp. $|v|=1)$. The set $D(M ; p)$ of all derivations at $p$ is then a graded left $Q$-module. If $v \in D(M ; p)$ and if $\phi=\left(x^{A}\right)$ is a local coordinate system around $p$, then the components $v^{A}$ of $v$ with respect to $\left(x^{A}\right)$ are defined by $v^{A} \doteq v\left(x^{A}\right)$. The maps $\left(\partial_{A}\right)_{p}: f \rightarrow\left(\partial_{A} f\right)(p)$ satisfy the relations (6.2) so that $\left(\partial_{A}\right)_{p} \in D(M ; p)_{A}$.

Proposition 6.1. For each local coordinate system $\phi=\left(x^{A}\right)$ around $p \in M$ the derivatives $\left(\partial_{A}\right)_{p}$ form up a left $Q$-module basis for $D(M ; p)$. Namely, for every $v \in D(M ; p)$ we have $v=v^{A}\left(\partial_{A}\right)_{p}$.

Proof. We first show that every derivation $v \in D(M ; p)$ annihilates germs of constant functions at $p$. Since $1^{2}=1$, it is evident that $v(1)=0$ and $Q_{0}$-linearity of $v$ implies that $v$ annihilates $Q_{0}$. Now for $a, b \in Q_{1}$ we have $a b \in Q_{0}$, and therefore $0=v(a b)=v(a) b+J^{|v|}(a) v(b)=v(a) b-v(b) a$ for all $a, b \in Q_{1}$. On the other hand $Q_{0}$-linearity of $v$ implies (Proposition 3.1, vi)) $v(a) b=v(b) a$ so that $v(a) b=0$ and by Proposition 3.1., i) we get $v(a)=0$. Now we make use of Lemma 5.1 and write in an open neighbourhood of $x_{0}=\phi(p)$

$$
f=\left(f \circ \phi^{-1}\right) \circ \phi=f(p)+\left(x^{A}-x_{0}^{\prime A}\right) g_{G} \circ \phi .
$$

By applying $v$ to both sides we get

$$
v(f)=v\left(x^{A}\right) g_{A}\left(x_{0}\right)=v^{A}\left(\partial_{A} f\right)(p) .
$$

Corollary 6.1. If $\left(x^{A}\right)$ and $\left(x^{A^{\prime}}\right)$ are local coordinate systems around $p \in M$, then

$$
v^{A^{\prime}}=v^{A} \frac{\partial x^{A^{\prime}}}{\partial x^{A}}(x(p))
$$

for each $v \in D(M ; p)$. In particular the graded left $Q$-modules $D(M ; p)$ and $\widetilde{T}(M ; p)$ are isomorphic.

Proof. Apply the above Proposition to $f=x^{A^{\prime}}$.

The isomorphism of $\tilde{T}(M ; p)$ and $D(M ; p)$ have been shown by referring to coordinate maps. It is of some interest to see how a coordinate free description can be given. If $v \in T(M ; p)$ then $v$ determines the even derivation $v$ by

$$
v:\left.f \mapsto \frac{d}{d t}(f \circ c)\right|_{t=0},
$$

where $c$ is a path through $p$ to which $v$ is tangent. Suppose now $\bar{v} \in \bar{T}(M ; p)$ is an odd tangent vector at $p$. Then, given $\varepsilon \in Q_{1}$, we have $\varepsilon \bar{v} \in T(M ; p)$, and for each $f \in Q_{p}(M)$ there exists a unique element $\bar{v}(f) \in Q$ such that $(\varepsilon \bar{v})(f)=\varepsilon \bar{v}(f)$ for all $\varepsilon \in Q_{1}$. The map $f \mapsto \bar{v}(f)$ so defined is then an odd derivation at $p$. Equivalently one may consider classes of equivalence of supersmooth maps $\bar{c}: \varepsilon \mapsto \bar{c}(\varepsilon)$ from open neighbourhoods of $0 \in Q_{1}$ to $M$, and define an odd tangent vector as such an equivalence class, so that for $\bar{v}=[\bar{c}]$ we have $\bar{v}^{A}=\left(d \bar{c}^{A} / d \varepsilon\right)_{\varepsilon=0}$. Such a point of view is however of little use. The reason is that while an even vector superfield 
generates one-parameter group of local, supersmooth, diffeomorphisms of $M$, an odd vector superfield can not be integrated even if one thinks about using an anticommuting parameter $\varepsilon \in Q_{1}$. The folk saying that one can integrate an odd vector field with a help of an anticommuting parameter is misleading, and what one usually does is an integration of an even vector field $\varepsilon \bar{V}, \varepsilon \in Q_{1}$ being fixed. One may say that odd vectors point out in directions which are complementary to $M$, what is precisely the case if $M$ is interpreted as the zero submanifold of the vector bundle $\widetilde{T}(M)$.

Having defined tangent bundle, the rest of differential geometry of supermanifolds is straightforward to develop. An even differential form at $p$ is an element of $T(M ; p)^{\dagger}{ }_{0}$ and a graded differential form at $p$ is an element of $T(M ; p)^{\dagger}=$ $L_{L}(T(M ; p) ; Q)$. Given a coordinate system $\phi=\left(x^{A}\right)$ around $p$, the differential forms $\left(d x^{A}\right)_{p}$ defined by $\left(d x^{A}\right)_{p}(v) \doteq v^{A}, v \in T(M ; p)$, form up a right $Q$-module basis for $T(M ; p)^{\dagger}$. The concepts of exterior derivative and exterior product can be defined, first for sections of $T(M ; p)^{\dagger}{ }_{0}$ evaluated on $T(\mathrm{M} ; p)$, then the extension procedure described in Sect. 4 can be applied at each point $p \in M$. The same method applies to Lie derivatives, connections etc.

\section{Supersymmetric Superspaces}

Let $(E, \Phi)(\operatorname{resp} .(F, \Psi))$ be a vector superspace of dimension $(4,0)($ resp. $(0,4))$ endowed with a complete family of coordinate maps $\phi: E \rightarrow\left(Q_{0}\right)^{4}$ (resp. $\psi: F \rightarrow$ $\left.\left(Q_{1}\right)^{4}\right)$, where $Q$ is a fixed BG-algebra over $\mathbb{R}$. Let $g$ (resp. $C$ ) be an even symmetric $Q_{0}$-bilinear form on $E$ (resp. on $F$ ). The form $g($ resp. $C$ ) considered as a map $g: E \rightarrow E^{\dagger}{ }_{0}$ (resp. $C: F \rightarrow F^{\dagger}{ }_{0}$ ) is assumed to be a bijection. The body of $g$ (see Sect. 4) is assumed to have signature $(-1,+1,+1,+1)$.

The two structures $(E, g)$ and $(F, C)$ are soldered by requiring that there is also given a map $\gamma \in L(E ; L(F ; F))$ such that

(i) $\gamma(v) \gamma(w)+\gamma(w) \gamma(v)=2\langle v, w \mid g\rangle 1_{F}, \quad v, w \in E$,

(ii) $C \gamma(v)=-\gamma(v)^{\dagger} C$ for all $v \in E$.

The map $v^{\dagger} \mapsto-C \gamma\left(g^{-1}\left(v^{\dagger}\right)\right)$ from $E_{0}^{\dagger}$ to $L\left(F ; F_{0}^{\dagger}\right)$ can be also interpreted as an element $\Omega \in L^{2}(F ; E)$ (see Proposition 4.1. (iii)), so that

$$
v^{\dagger}(\Omega(y, z))=-\left(\left(C \gamma\left(g^{-1}\left(v^{\dagger}\right)\right)\right)(z)\right)(y)
$$

for all $y, z \in F, v^{\dagger} \in E^{\dagger}$. The relation (ii) implies then that $\Omega$ is skewsymmetric.

Let $S$ be an affine space over $F$. Each tangent space $T\left(S ; \theta_{0}\right)$ is canonically isomorphic to $F$ and $\Omega$ can be also interpreted as an $E$-valued 2 -form on $S$. Let $M$ be a supersmooth principal bundle over $S$ with $(E,+)$ as the structure group and bundle projection $\Pi: M \rightarrow S$. For each $v \in E$ let $T_{v}$ denotes the translation $T_{v}: M \rightarrow M$ so that $\Pi \circ T_{v}=\Pi$. The form $\Omega$ is now lifted to $M$ by $\Pi$ and denoted with the same letter $\Omega$. It is horizontal (i.e. $\Omega(X, Y)=0$ if $d \Pi(X)=0$ ) and closed (i.e. $d \Omega=0$ ). Since the structure group is Abelian, $\Omega$ can be considered as a curvature 2-form of principal connection $\omega$.

Definition 7.1. Supersymmetric superspace $M$ is a supersmooth trivializable 
principal bundle $(M, \Pi, S, E)$ equipped with a principal connection $\omega$ such that $d \omega=\Omega$. A symmetry of $M$ is a vector superfield $X$ which preserves the structure of $M$, i.e.

(a) $X$ is invariant vector field, i.e.

$$
(d \Pi)\left(X\left(T_{v} p\right)\right)=d \Pi(X(p))
$$

for all $p \in M$ and $v \in E$. Equivalently, $X$ preserves the fibering of $M$, i.e. there exists vector superfield $X^{\prime}$ on $S$ such that $X \stackrel{\pi}{\sim} X^{\prime}$.

(b) $X$ generates isometries between the fibers of $M$, i.e.

$$
X(\langle Y, Z \mid g\rangle)=\left\langle\mathscr{L}_{X} Y, Z \mid g\right\rangle+\left\langle Y, \mathscr{L}_{X} Z \mid g\right\rangle
$$

for all vertical vector superfields $Y, Z: M \rightarrow T(M)$, where $g$ is the induced metric on vertical subspaces.

(c) $X$ transforms horizontal subspaces into horizontal ones, i.e.

$$
\left\langle\mathscr{L}_{X} Y \mid \omega\right\rangle=0 \text { if }\langle Y \mid \omega\rangle=0 .
$$

(d) $X^{\prime}$ is an isometry of $S$ endowed with the induced metric $C$.

To describe explicitly all symmetries (which have been defined without any reference to coordinate systems) one has to decide which coordinate systems are the convenient ones. A strategy of adapting a coordinate system can be described as follows.

1. Chose a preferred set of real $\gamma$-matrices $\gamma_{\mu \beta}^{\alpha}$ satisfying $\left[\gamma_{\mu}, \gamma_{\nu}\right]_{+}=2 \eta_{\mu \nu}$ with $\left(\eta_{\mu v}\right)=\operatorname{diag}(-1,+1,+1,+1)$, (or use $i \gamma_{\mu}$ if $\gamma_{\mu}$ are chosen imaginary), and choose a real skew-symmetric matrix $\left(C_{\alpha \beta}\right)$ (charge conjugation matrix) so that the matrix equation $C \gamma_{\mu} C^{-1}=-\gamma_{\mu}{ }^{T}$ holds. A straightforward argument shows that there exist $Q_{0}$-module bases $e_{\mu} \in E, \bar{e}_{\alpha} \in \bar{F}$ such that $\left\langle e_{\mu}, e_{v} \mid g\right\rangle=\eta_{\mu v},\left\langle\bar{e}_{\alpha}, \bar{e}_{\beta} \mid C\right\rangle=C_{\alpha \beta}$ and $\gamma\left(e_{\mu}\right) \bar{e}_{\alpha}=\bar{e}_{\beta} \gamma_{\mu \alpha}{ }^{\beta}$. The form $\Omega \in L^{2}(F ; E)$ can then be written as $\Omega\left(\bar{e}_{\alpha}, \bar{e}_{\beta}\right)^{\mu}=-\gamma_{\alpha \beta}^{\mu \beta}$, where $\gamma_{\alpha \beta}^{\mu}=C_{\alpha \delta} \eta^{\mu \nu} \gamma_{v \beta}^{\delta}$.

2. Choose $\theta_{0} \in S$ so that $S$ can be identified with $F$, and for every $\theta \in S$ one has $\theta=\theta_{0}+\theta^{\alpha} \bar{e}_{\alpha}$ with $\theta^{\alpha} \in Q_{1}$.

3. Having already chosen coordinate systems on the structure group $E$ and on the base manifold $S$, there remains to chose a supersmooth section of $M$. The explicit form of the connection 1-form $\omega$ will depend on this choice. A convenient choice of such a section is the one in which $\omega$ can be written as

$$
\omega^{\mu}=d x^{\mu}+\frac{1}{2} d \theta^{\alpha} \gamma_{\alpha \beta}^{\mu} \theta^{\beta}
$$

and it is easy to see that such a choice is always possible.

Now in an adapted coordinate system the most general symmetry $X$ can be calculated. The result reads

$$
X=b^{\mu} \partial_{\mu}+a^{\alpha}\left(\partial_{\alpha}+\frac{1}{2} \gamma_{\alpha \beta}^{\mu} \theta^{\beta} \partial_{\mu}\right)+\frac{1}{2} b^{\mu v}\left(x_{\mu} \partial_{v}-x_{v} \partial_{\mu}-\sum_{\mu \nu \beta}^{\alpha} \theta^{\beta} \partial_{\alpha}\right),
$$

where $b^{\mu}, b^{\mu \nu}=-b^{\nu \mu}$ are from $Q_{0}, a^{\alpha} \in Q_{1}$, and $\Sigma_{\mu \nu} \doteq \frac{1}{4}\left[\gamma_{\mu}, \gamma_{\nu}\right]$.

There is a straightforward generalization of the above construction which leads to an extended supersymmetry. The vector superspace $F$ is to be replaced by $F^{(N)}=\mathbb{R}^{N} \otimes F$, and $C$ is replaced by $C^{(N)}=I \otimes C$ so that $C^{(N)}: \mathbb{R}^{N} \otimes F \rightarrow \mathbb{R}^{N} \otimes F^{\dagger}$ if $\mathbb{R}^{N}$ is identified with its dual. Coordinates in $M^{(N)}$ are now $\left(x^{\mu}\right)$ and 
$\left(\theta^{\alpha i}\right)(\mu=1, \ldots, 4 ; i=1, \ldots, N)$, and in an adapted coordinate system curvature and connection forms read

$$
\begin{aligned}
& \Omega^{(N)}=-\frac{1}{2} d \theta^{\alpha i} \wedge d \theta^{\beta j} \delta_{i j} \gamma_{\alpha \beta}^{\mu} \partial_{\mu}, \\
& \omega^{(N)}=d x^{\mu}+\frac{1}{2} d \theta^{\alpha i} \gamma_{\alpha \beta}^{\mu} \delta_{i, j} \theta^{\beta j} .
\end{aligned}
$$

The most general symmetry $X$ takes the form

$$
\begin{aligned}
X= & b^{\mu} \partial_{\mu}+a^{\alpha i}\left(\partial_{\alpha i}+\frac{1}{2} \gamma_{\alpha \beta}^{\mu} \delta_{i j} \theta^{\beta j} \partial_{\mu}\right) \\
& +\frac{1}{2} b^{i j}\left(\theta^{\alpha k} \delta_{k i} \partial_{\alpha j}-\theta^{\alpha k} \delta_{k j} \partial_{\alpha i}\right) \\
& +\frac{1}{2} b^{\mu \nu}\left(x_{\mu} \partial_{v}-x_{v} \partial_{\mu}-\sum_{\mu \nu} \alpha \theta^{\beta i} \partial_{\alpha i}\right),
\end{aligned}
$$

with $a^{\alpha i} \in Q_{1}$ and $b^{\mu}, b^{\mu v}=-b^{v \mu}, b^{i j}=-b^{j i}$ from $Q_{0}$.

In both cases (i.e. of simple and extended supersymmetry) a real manifold $\tilde{M}$ can be defined as the body of $M$. In both cases $\tilde{M}$ is just an ordinary Minkowski space. In the last example given below the quotient manifold will have a structure of a principal bundle Minkowski space with 0(2) as its structure group.

To describe superspace with central charge (see [5], and references there) let $\mathbb{R}^{2}$ be endowed with its canonical metric $\delta=\left(\delta_{i j}\right), i, j=1,2$, and let $T$ denote the canonical representation of $0(2)$ on $\mathbb{R}^{2}$. We define $F_{c}=\mathbb{R}^{2} \otimes F$ and equip it with the metric $C_{c}=\delta \otimes C$ as in the last example. $S_{c}$ is an affine space over $F_{c}$. Now the supergroup $0^{\prime}(2)$ has to be defined. We define $0^{\prime}(2)$ as $0(2) \times Q_{0}^{\prime}$ with the group multiplication $(q, a)\left(q^{\prime}, a^{\prime}\right) \doteq\left(q q^{\prime}, a+a^{\prime}\right)$ for $q, q^{\prime} \in 0(2), a, a^{\prime} \in Q_{0}^{\prime}$.

Let for each $\phi \in \mathbb{R}, T(\phi)$ denotes the matrix

$$
T(\phi) \doteq\left(\begin{array}{rr}
\cos \phi, & \sin \phi \\
-\sin \phi, & \cos \phi
\end{array}\right) .
$$

Then, given $\bar{\phi} \in \mathbb{R}$, we have a coordinate map with domain $0(2) \backslash T(\bar{\phi})$ and range $(0,2 \pi) \subset \mathbb{R}$ given by $T(\phi+\bar{\phi}) \mapsto \phi$. We define the local coordinate map $x^{5}$ for $0^{\prime}(2)$ with domain $(0(2) \backslash T(\bar{\phi})) \times Q_{0}^{\prime}$ and range $\sigma^{-1}((0,2 \pi)) \subset Q_{0}$ by $x^{5}:(T(\phi), a) \mapsto$ $\phi+a$.

It is evident that with the above definition $0^{\prime}(2)$ is a superanalytic super-group according to [6], with its Lie algebra isomorphic to $Q_{0}$. The representation $T$ of $0(2)$ on $\mathbb{R}^{2}$ extends by analyticity to a representation of $0^{\prime}(2)$ on $F_{c}$.

Let now $E^{5}=0^{\prime}(2) \times E$ so that $E^{5}$ is also a supergroup with its Lie algebra $\varepsilon^{5}$ isomorphic to $Q_{0} \oplus E$. On $Q_{0} \oplus E$ we have the invariant metric $g^{5}=1 \oplus g$. $M^{5}$ is defined to be a principal fibre bundle over $S_{c}$ with $E^{5}$ as its structure group.

On $F_{c}$ we have now representation $\Gamma=T \otimes \gamma$ of $E^{5}$ and the derived representation $d \Gamma=d T \otimes 1_{F}+1_{\mathbb{R}^{2}} \otimes \gamma$ of $\varepsilon^{5}$. The form $\Omega$ is defined as in the first example but with $C, \gamma, g$ replaced by $C_{c}, d \Gamma, g^{5}$. In an adapted coordinate system we then have

$$
\begin{aligned}
& \Omega=-\frac{1}{2} d \theta^{\alpha i} \wedge d \theta^{\beta j}\left(\gamma_{\alpha \beta}^{\mu} \delta_{i j} \frac{\partial}{\partial x^{\mu}}+C_{\alpha \beta} \varepsilon_{i j} \frac{\partial}{\partial x^{5}}\right), \\
& \omega^{\mu}=d x^{\mu}+\frac{1}{2} d \theta^{\alpha i} \gamma_{\alpha \beta}^{\mu} \theta^{\beta j} \delta_{i j}, \\
& \omega^{5}=d x^{5}+\frac{1}{2} d \theta^{\alpha i} C_{\alpha \beta} \theta^{\beta j} \varepsilon_{i j} .
\end{aligned}
$$


Looking for the symmetries of $M^{5}$ which preserve all the relevant structure (including the direct sum structure of vertical subspaces) we can find the following generators:

$$
\begin{aligned}
P_{\mu} & =\frac{\partial}{\partial x^{\mu}}, \quad Z=\frac{\partial}{\partial x^{5}}, \\
M_{\mu \nu} & =x_{\mu} \partial_{\nu}-x_{\nu} \partial_{\mu}-\sum_{\mu \nu \beta}^{\alpha} \theta^{\beta i} \frac{\partial}{\partial \theta^{\alpha i}}, \quad B=\varepsilon^{i j} \theta^{\alpha k} \delta_{k i} \frac{\partial}{\partial \theta^{\alpha j}}, \\
Q_{\alpha i} & =\frac{\partial}{\partial \theta^{\alpha i}}+\frac{1}{2} \gamma_{\alpha \beta}^{\mu} \delta_{i j} \theta^{\beta j} \frac{\partial}{\partial x^{\mu}}+\frac{1}{2} C_{\alpha \beta} \varepsilon_{i j} \theta^{\beta j} \frac{\partial}{\partial x^{5}} .
\end{aligned}
$$

The interesting commutation relations are

$$
\begin{aligned}
{\left[Q_{\alpha i}, Q_{\beta j}\right]_{+} } & =\gamma_{\alpha \beta}^{\mu} \delta_{i j} P_{\mu}+C_{\alpha \beta} \varepsilon_{i j} Z, \\
{\left[Q_{\alpha i}, B\right] } & =\delta_{i j} \varepsilon^{j k} Q_{\alpha k} .
\end{aligned}
$$

Remark 7.1. If $Q_{0}$-module bases $e_{\mu} \in E, \bar{e}_{\alpha i} \in \bar{F}_{c}$ are chosen, then the global vielbein on $M^{5}$ is defined by the horizontal lift of $\bar{e}_{\alpha i}-s$, so that $M^{5}$ is endowed with a teleparallelism. The affine connection coefficients can be easily calculated, the only non-vanishing being $\Gamma_{\alpha i, \beta j}^{\mu}=(1 / 2) \gamma_{\alpha \beta}^{\mu} \delta_{i j}, \Gamma_{\alpha i, \beta j}^{5}=(1 / 2) C_{\alpha \beta} \varepsilon_{i j}$.

Remark 7.2. Since in all three examples the supersymmetric superspaces are principal bundles endowed with principal connections, we have covariant derivatives $D_{\alpha i}$ which can act on differential forms on the bundle to produce horizontal ones (see e.g. [7], Chap. VI., Sect. 4). In particular, when applied to functions on $M$, they coincide with the "covariant derivatives" exploited by Salam and Strathdee [8].

Acknowledgement. One of the authors (A. J.) would like to thank Professor R. Hagg for his interest, critical discussion, and hospitality extended at II. Institut für Theoretische Physik, Universität Hamburg, where this work was completed.

\section{Reference}

1. Rogers, A.: J. Math. Phys. 21, 1352 (1980)

2. Jadczyk, A., Pilch, K.: to appear

3. Dieudonné, J.: Foundations of modern analysis. New York, London: Academic Press 1960

4. Lang, S. : Differential manifolds. Reading, MA: Addision-Wesley Publishing Company, Inc., 1972

5. Haag, R., Lopuszanski, J., Sohnius, M.: Nucl. Phys. B88, 255-274 (1975)

6. Rogers, A.: Super lie groups: Global topology and local structure. Preprint ICTP/79-80/15

7. Greub, W., Halperin, S., Vanston, R.: Connections, curvature, and cohomology. Vol. II. New York, London: Academic Press 1973

8. Salam, A., Strathdee, J.: Phys. Rev. D11, 1521-1535(1975)

Communicated by R. Haag

Received July 14, 1980 